Research Paper

\title{
An Increased Neutrophil-to-Lymphocyte Ratio Predicts Incomplete Response to Therapy in Differentiated Thyroid Cancer
}

\author{
Fang Lee 1, Po-Sheng Yang 1, Ming-Nan Chien 2, Jie-Jen Lee 1, Ching-Hsiang Leung ${ }^{2}$, Shih-Ping Cheng 1,3 \\ 1. Department of Surgery, MacKay Memorial Hospital and Mackay Medical College, Taipei, Taiwan \\ 2. Division of Endocrinology and Metabolism, Department of Internal Medicine, MacKay Memorial Hospital and Mackay Medical College, Taipei, Taiwan \\ 3. Department of Pharmacology, School of Medicine, College of Medicine, Taipei Medical University, Taipei, Taiwan
}

$\square$ Corresponding author: Shih-Ping Cheng, MD, PhD. Department of Surgery, MacKay Memorial Hospital, 92, Section 2, Chung-Shan North Road, Taipei 10449, Taiwan. Phone: +886 22543 3535, Fax: +886 22723 3897, E-mail: surg.mmh@gmail.com

(c) Ivyspring International Publisher. This is an open access article distributed under the terms of the Creative Commons Attribution (CC BY-NC) license (https:// creativecommons.org/licenses/by-nc/4.0/). See http://ivyspring.com/terms for full terms and conditions.

Received: 2018.07.13; Accepted: 2018.09.22; Published: 2018.11.23

\begin{abstract}
Background: Previously we have shown that an elevated baseline neutrophil-to-lymphocyte ratio (NLR) was associated with a high risk of recurrence in patients with differentiated thyroid cancer. The clinical significance of the longitudinal changes in NLR following treatment remained unestablished.

Methods: Adults patients with differentiated thyroid cancer were included in the study if the follow-up NLR data at 6 to 18 months after initial treatment were available. The response to treatment was categorized as excellent, indeterminate, biochemical incomplete, and structural incomplete as per guidelines of the American Thyroid Association.

Results: Among 151 patients with thyroid cancer, a significant decrease in NLR following treatment was observed in those with stage I disease, those with low risk of recurrence, and those with an excellent response to therapy. Patients with a structural incomplete response had a significant increase in NLR at follow-up $(p=0.012)$. On multivariate analysis, incomplete response to therapy was associated with male sex (odds ratio $[O R]=3.35)$, tumor size $(O R=1.63)$, lymph node metastasis $(O R=4.80)$, distant metastasis $(O R=12.95)$, and increased NLR $(O R=13.68)$.

Conclusions: An increase in systemic inflammation following treatment as measured by NLR is independently associated with an incomplete response to therapy in differentiated thyroid cancer.
\end{abstract}

Key words: Neutrophil-to-lymphocyte ratio; Dynamic risk stratification; Differentiated thyroid cancer; Inflammation

\section{Introduction}

Tumor-associated inflammation has been considered as an emerging hallmark of cancer [1]. Chronic inflammation may elicit neoplastic development, progression, and metastatic dissemination. On the other hand, the acquisition and accumulation of genetic alterations, as well as the loss of normal physiological regulation, may induce the expression of tumor-associated antigens. Malignant transformation is therefore accompanied by inflammatory responses resulting from insufficient or inappropriate activation of innate and adaptive immunity. These inflammatory responses may paradoxically promote tumorigenesis and can have opposing effects on treatment efficacy [2]. In this context, inflammatory biomarkers may aid in the prediction of patient outcome and the assessment of treatment response [3].

The peripheral blood neutrophil-to-lymphocyte ratio (NLR) is an inexpensive and widely available biomarker of the host immune system. An elevated NLR has been suggested to be related to poor prognosis in various solid cancers [4]. Previously, we 
have shown that an elevated preoperative NLR was associated with a high risk of recurrence in patients with differentiated thyroid cancer [5]. Subsequent studies confirmed that higher NLR values may be associated with a more aggressive tumor profile $[6,7]$. These observations suggest that although differentiated thyroid cancer is a relative indolent malignancy with an excellent prognosis, the phenomenon of tumor-promoting inflammation is also present in thyroid cancer patients.

Despite a great interest in the prognostic role of NLR and other inflammatory biomarkers in cancer patients, considerably less attention has been given to the dynamics of post-treatment changes in these biomarkers. Furthermore, the conventional staging and risk stratification systems based on the clinicopathological information at diagnosis may not predict persistent disease and recurrence accurately. The outcome of cancer patients is closely related to the response to treatment. It has been proposed that the initial risk estimates should be actively modified over time based on the response to therapy and the course of the disease [8]. The purpose of the present study was to correlate the longitudinal NLR changes with the response to treatment in differentiated thyroid cancer. The results of this study may yield further insights into the possible link between inflammatory markers and the disease status in thyroid cancer.

\section{Patients and Methods}

Following approval of by the Institutional Review Board (17MMHIS035), a review of the prospectively maintained endocrine surgery registry was conducted to identify all thyroid surgeries performed at MacKay Memorial Hospital, Taipei, Taiwan, from July 2013 to December 2015. All adult patients undergoing thyroidectomy were eligible according to the inclusion criteria. The exclusion criteria were: major immunomodulatory conditions (including immunodeficiency and active infection) or chronic kidney disease stage 3 or greater $(n=25)$, operation for recurrent thyroid cancer $(\mathrm{n}=16)$, non-differentiated thyroid cancer $(\mathrm{n}=7)$, and age younger than 20 years $(n=6)$.

All procedures were performed under general anesthesia. The complete blood count and leukocyte differential count were routinely measured one day before surgery. The NLR was calculated by dividing the absolute neutrophil count by the lymphocyte count [9]. The follow-up NLR data were obtained, if available, at 6 to 18 months after treatment when the patients were taking thyroxine suppression or replacement therapy. The difference in the NLR $(\triangle N L R)$ was calculated as the NLR at follow-up minus the NLR before surgery. The standard deviation of the
NLR distribution was determined for the study cohort. Increased NLR was defined as an NLR increase greater than or equal to the standard deviation [10]. Decreased NLR was defined as an NLR decrease more than or equal to the standard deviation. Otherwise, the patients were considered to have a stable NLR.

Patients with differentiated thyroid cancer underwent either lobectomy or total thyroidectomy and, if indicated, therapeutic central and/or lateral neck lymph node dissection. Unilateral prophylactic central neck dissection was performed in clinically node-negative patients [11]. The decision to have radioactive iodine (RAI) therapy was at the discretion of treating physicians on the basis of clinical need. Patient management was regularly discussed at multidisciplinary team meetings. Thereafter, patients were followed up with periodic clinical examinations, biochemical tests, and imaging studies [12].

The tumor staging upon initial treatment was based on the seventh edition of the American Joint Committee on Cancer/Union for International Cancer Control tumor-node-metastasis (TNM) system. The mutational status of $B R A F$ was routinely determined by Sanger sequencing on cytological cell blocks or surgical specimens [13]. The risk of recurrence was evaluated using the modified American Thyroid Association (ATA) initial risk stratification system, which considers low-volume lymph nodal metastases as low risk [14]. Patients who had a total thyroidectomy and RAI therapy were reclassified by the response to the initial therapy as per the 2015 ATA guidelines [14]. In our institute, the response to therapy was typically assessed using thyroidstimulating hormone (TSH)-stimulated thyroglobulin (Tg) values obtained 6 to 18 months after initial therapy. The restratification scheme included the results of imaging studies (neck ultrasound with or without RAI scintigraphy) and stimulated or suppressed $\mathrm{Tg}$ and anti-Tg antibody levels during the follow-up period and divided the patients into four categories: excellent, indeterminate, biochemical incomplete, and structural incomplete response. For those who underwent lobectomy or total thyroidectomy without RAI therapy, the response to therapy was evaluated using the dynamic risk stratification proposed by Momesso and colleagues [15].

Continuous variables are presented as medians and interquartile ranges (IQRs), while categorical variables are presented as numbers and percentages. The TNM stage, ATA risk of recurrence, and the response to therapy were categorized as ordinal variables. Differences in medians between two groups were compared with nonparametric Mann-Whitney U 
tests. The Wilcoxon rank signed tests were used to assess NLR differences before and after treatment [16]. Trends were detected using Spearman's nonparametric correlation analysis. Logistic regression analysis was performed to determine the odds ratios (ORs) of variables with $95 \%$ confidence intervals (CIs) for an incomplete (biochemical or structural) response to therapy. Multivariate analysis using stepwise logistic regression was conducted on parameters shown to be significant in the univariate analysis. All statistical tests were two-sided at the $5 \%$ significance level. Analyses were performed using Stata statistical software (version 14.0; StataCorp, College Station, TX, USA).

\section{Results}

A total of 587 patients met the inclusion and exclusion criteria. Among these patients, 357 (61\%) underwent thyroidectomy for benign thyroid lesions and $230(39 \%)$ for differentiated thyroid cancer. Benign thyroid lesions included nodular goiter $(\mathrm{n}=$ 252), follicular adenoma $(\mathrm{n}=53)$, Hashimoto's thyroiditis $(\mathrm{n}=28)$, and Graves' disease $(\mathrm{n}=24)$. During the follow-up period, $87(24 \%)$ patients with benign lesions and 151 (66\%) patients with differentiated thyroid cancer had available NLR data for analysis. These patients constituted the study cohort (Table 1). At a median follow-up of 19 months, 10 patients had locoregional recurrence (neck lymph nodes, $\mathrm{n}=9$; thyroid bed, $\mathrm{n}=1$ ), and one patient died of locally advanced disease and lung metastases. Two additional patients died of irrelevant diseases (cervical cancer and sepsis, respectively).

Before treatment, the median NLR was 1.93 (IQR, 1.53-2.61) and 1.96 (IQR, 1.51-2.59) for the benign and cancer groups, respectively $(p=0.633)$. In the benign group, 72 patients had thyroidectomy for nodular goiter and 15 for follicular adenoma. As shown in Figure 1, there was no significant change in NLR after thyroidectomy $(p=0.113)$.

Among 151 patients in the cancer group, the majority $(\mathrm{n}=145,96 \%)$ were treated for papillary thyroid cancer, while 6 patients had follicular thyroid cancer. Interestingly, the overall NLR significantly decreased to 1.78 (IQR, 1.34-2.48) following treatment $(p=0.037)$. The difference was only significant in the total thyroidectomy plus RAI therapy group $(\mathrm{n}=128$, $p=0.020)$ but not in the lobectomy group $(\mathrm{n}=14, p=$ $0.778)$ or total thyroidectomy only group $(\mathrm{n}=9, p=$ 0.678). In parallel to the decrease in NLR, the median total white blood cell count decreased from 7100 before surgery to 6300 at follow-up $(p<0.001)$. There was no difference in hemoglobin levels before and after treatment $(p=0.845)$.
Table 1. Patient characteristics

\begin{tabular}{|c|c|}
\hline & Study cohort $(\mathrm{n}=151)$ \\
\hline Female & $124(82 \%)$ \\
\hline Age (years) & $47(37-56)$ \\
\hline Body mass index $\left(\mathrm{kg} / \mathrm{m}^{2}\right)$ & $23.4(21.2-27.7)$ \\
\hline Hashimoto's thyroiditis & $26(17 \%)$ \\
\hline Papillary thyroid cancer & $145(96 \%)$ \\
\hline$B R A F$ mutation & $112(74 \%)$ \\
\hline \multicolumn{2}{|l|}{ Treatment } \\
\hline Lobectomy & $14(9 \%)$ \\
\hline Total thyroidectomy & $9(6 \%)$ \\
\hline Total thyroidectomy + RAI & $128(85 \%)$ \\
\hline Tumor size $(\mathrm{cm})$ & $1.8(1.2-2.5)$ \\
\hline \multicolumn{2}{|l|}{ Extrathyroidal extension } \\
\hline No & $77(51 \%)$ \\
\hline Microscopic & $58(38 \%)$ \\
\hline Macroscopic & $16(11 \%)$ \\
\hline Multifocality & $41(27 \%)$ \\
\hline Lymphovascular invasion & $50(33 \%)$ \\
\hline \multicolumn{2}{|l|}{ Lymph node metastasis } \\
\hline No & $70(46 \%)$ \\
\hline N1a & $68(45 \%)$ \\
\hline N1b & $13(9 \%)$ \\
\hline Distant metastasis & $10(7 \%)$ \\
\hline \multicolumn{2}{|l|}{ TNM stage } \\
\hline Stage I & $85(56 \%)$ \\
\hline Stage II & $7(5 \%)$ \\
\hline Stage III & $36(24 \%)$ \\
\hline Stage IV & $23(15 \%)$ \\
\hline \multicolumn{2}{|l|}{ ATA risk of recurrence } \\
\hline Low risk & $39(26 \%)$ \\
\hline Intermediate risk & $87(58 \%)$ \\
\hline High risk & $25(17 \%)$ \\
\hline \multicolumn{2}{|l|}{ Response to therapy } \\
\hline Excellent & $92(61 \%)$ \\
\hline Indeterminate & $25(17 \%)$ \\
\hline Biochemical incomplete & $22(15 \%)$ \\
\hline Structural incomplete & $12(8 \%)$ \\
\hline
\end{tabular}

Note: Data are given as frequencies (percentages) or medians (interquartile ranges). Abbreviations: ATA, American Thyroid Association; RAI, radioactive iodine.

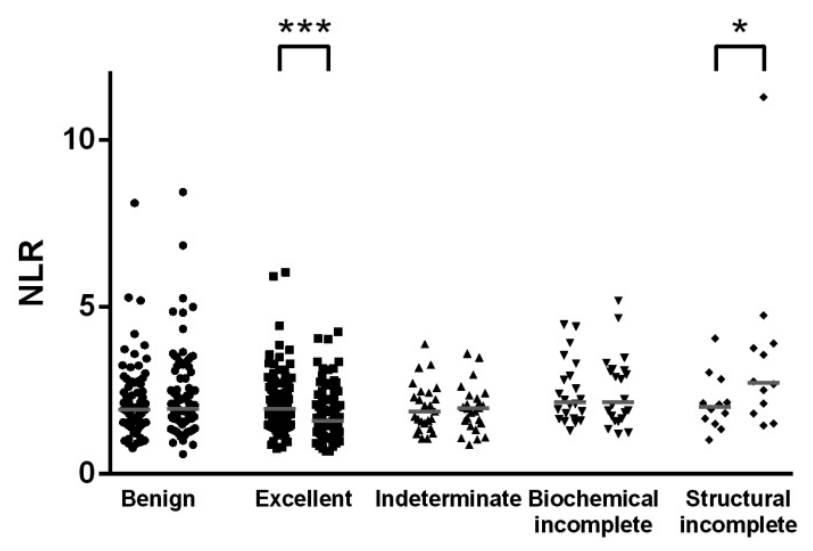

Figure 1. Scatter plots of preoperative (left dot groups) and post-treatment (right dot groups) values of the neutrophil-to-lymphocyte ratio (NLR) in patients with benign thyroid lesions $(n=87)$ and differentiated thyroid cancer categorized by the response to therapy (excellent, $n=92$; indeterminate, $n=$ 25 ; biochemical incomplete, $n=22$; structural incomplete, $n=12$ ). *, Wilcoxon signed rank test $p<0.05 ; * * *, p<0.001$.

We further analyzed the changes in NLR among different subgroups. The significant decrease in NLR following treatment was observed in patients with stage I disease $(p=0.004)$ but not those with stage II to 
IV disease. Additionally, the significant decrease in NLR was observed in patients with low risk of recurrence $(p=0.014)$ but not those with intermediate risk $(p=0.051)$ or high risk $(p=0.412)$. Interestingly, we found a significant decrease in NLR among patients with an excellent response to therapy $(p<$ 0.001 , Figure 1). Of interest, 12 patients with a structural incomplete response had a significant increase in NLR from a median of 2.01 (IQR, 1.58-2.49) before surgery to 2.73 (IQR, 1.96-3.84) at follow-up ( $p$ $=0.012$ ).

The median $\triangle$ NLR in patients with differentiated thyroid cancer was -0.15 (IQR, -0.62 to 0.45 ), which was significantly different from that of the benign group (median, $+0.09 ; p=0.008$ ). The $\Delta \mathrm{NLR}$ was not associated with the TNM stage ( $p$ for trend $=0.098$ ). However, the $\triangle$ NLR was positively associated with the ATA risk of recurrence $(p$ for trend $=0.017$ ) and response to therapy ( $p$ for trend $<0.001$ ). Scatter plots are depicted in Figure 2.

The standard deviation of preoperative NLR was 1.08 in our cancer patients. By definition, $12(8 \%)$ patients with a $\triangle N L R \geq 1.08$ had an increased NLR, and $17(11 \%)$ with a $\triangle \mathrm{NLR} \leq-1.08$ had a decreased NLR. The remaining $81 \%$ of the patients had stable NLR following treatment. As shown in Figure 3, the proportions of patients having an increased NLR significantly increased in those with a higher risk of recurrence or incomplete response to therapy (both $p$ $<0.001)$.

To validate whether an increased NLR was an independent predictor for incomplete response to therapy, univariate and multivariate logistic regression analyses were performed. We found that male sex, larger tumor size, nodal metastasis, distant metastasis, and increased NLR independently predicted the risk of incomplete response to therapy (Table 2). When the increased NLR was replaced by the $\triangle \mathrm{NLR}$ as a continuous variable in the model, the results remained unchanged. Male sex (OR, 3.65, 95\% $\mathrm{CI}$, 1.13-11.77), tumor size (OR, 1.84, 95\% CI, 1.14-2.96), lymph node metastasis (OR, 5.90, 95\% CI, 1.55-22.49), distant metastasis (OR, 8.17, 95\% CI, 1.37-48.82), and the absolute change in NLR (OR, 3.04, 95\% CI, 1.49-6.19) were independent risk factors for incomplete response to therapy.

\section{Discussion}

Tumor-associated inflammatory cells are not uncommon in thyroid cancer on microscopic examination [17]. Various and distinct patterns of immune infiltrates may have different prognostic implications [18]. Immune dysregulation is one of the probable mechanisms involving in worse prognosis in older patients with papillary thyroid cancer [19].
Recently, we reported that the expression of interleukin-8 (IL-8) was progressively upregulated during disease progression [20]. IL-8 is a proinflammatory CXC chemokine which induces neutrophil chemotaxis and promotes angiogenesis. Contrary to anaplastic thyroid cancer, intratumoral neutrophil infiltration is rarely seen in the absence of necrosis. Nonetheless, neutrophil infiltration in differentiated thyroid cancer, when present, may portend an aggressive phenotype [21].

Generally, differentiated thyroid cancer is associated with a low degree of systemic inflammatory response. Based on our experience and review of the literature, a baseline NLR is not helpful to distinguish thyroid cancer from benign thyroid lesions [5, 22]. However, the peripheral blood NLR consistently showed a positive correlation with tumor size among patients with differentiated thyroid cancer [5-7]. It suggests that differentiated thyroid cancer exhibits a weak but distinct antigenicity which induces a certain level of systemic inflammatory response. Nonetheless, the baseline NLR appeared to have no association with disease-free survival in patients with differentiated thyroid cancer [6, 7]. This may be due to the fact that patient with differentiated thyroid cancer had relatively low NLR values compared with those of patients with other solid tumors [4], or that the analyses were underpowered to detect small differences in the context of the overall good prognosis of thyroid cancer.
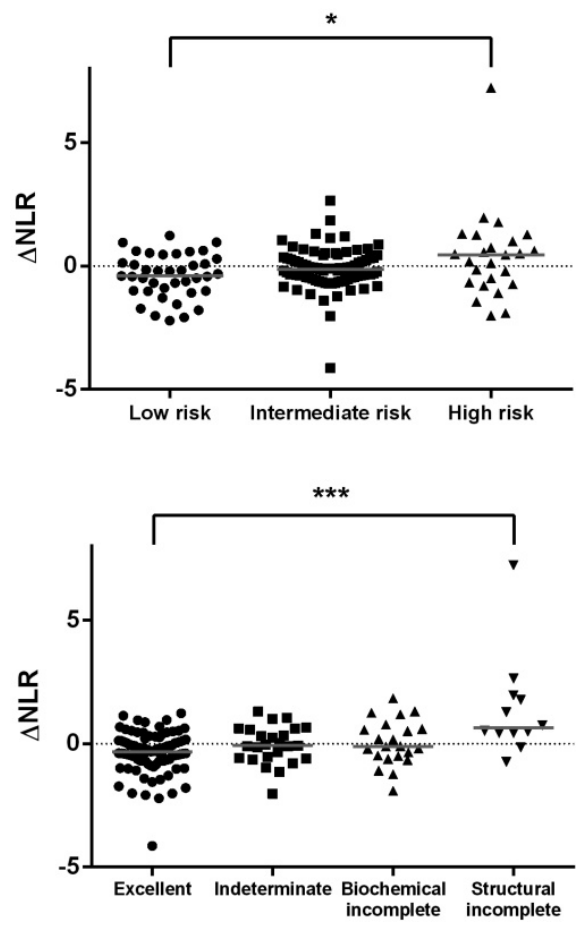

Figure 2. Scatter plots of the difference in the post-treatment and preoperative neutrophil-to-lymphocyte ratio $(\triangle N L R)$ in patients with differentiated thyroid cancer categorized by the risk of recurrence (upper panel) or response to therapy (lower panel). *, $p$ for trend $<0.05$; ***, $p<0.001$. 

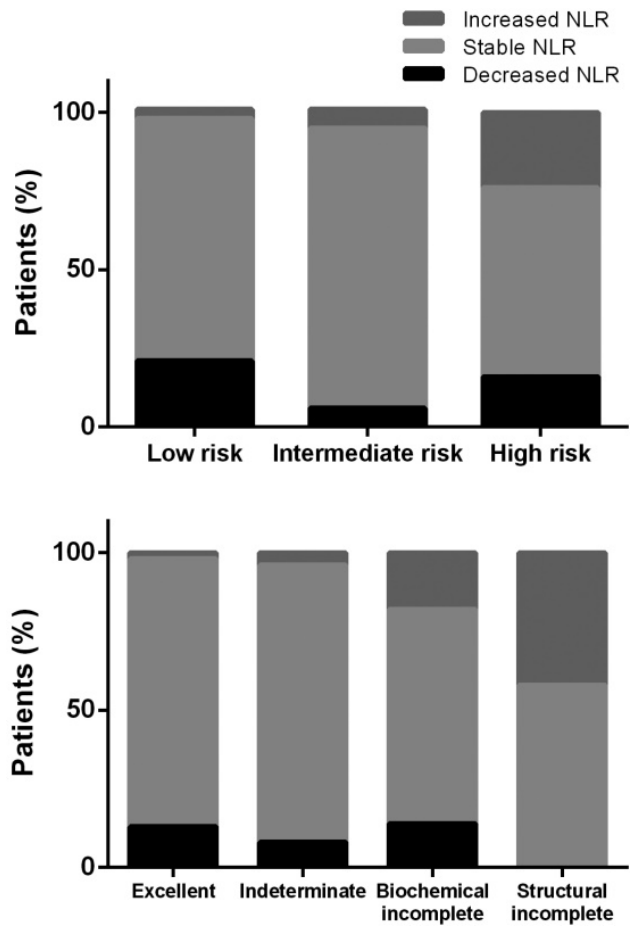

Figure 3. Percentages of the relative changes in the neutrophil-to-lymphocyte ratio (NLR) after treatment of differentiated thyroid cancer, categorized by the risk of recurrence (upper panel) or response to therapy (lower panel).

Table 2. Univariate and multivariate logistic regression analyses of factors predicting an incomplete response to therapy in differentiated thyroid cancer

\begin{tabular}{|c|c|c|c|c|c|c|}
\hline & \multicolumn{3}{|c|}{ Univariate } & \multicolumn{3}{|c|}{ Multivariate } \\
\hline & $\begin{array}{l}\text { Odds } \\
\text { ratio }\end{array}$ & $95 \% \mathrm{CI}$ & $p$-value & $\begin{array}{l}\text { Odds } \\
\text { ratio }\end{array}$ & $95 \% \mathrm{CI}$ & $p$-value \\
\hline Age ${ }^{\dagger}$ & 1.010 & $0.987-1.032$ & 0.396 & & & \\
\hline Male sex & 3.348 & $1.620-6.921$ & 0.001 & 3.593 & $\begin{array}{l}1.133- \\
11.394\end{array}$ & 0.030 \\
\hline
\end{tabular}

Body mass index $1.056 \quad 0.977-1.143 \quad 0.171$

Hashimoto's $\quad 0.631 \quad 0.248-1.606 \quad 0.334$

thyroiditis

$\begin{array}{lllllll}\text { Tumor size }{ }^{\dagger} \quad 1.629 & 1.258-2.110<0.001 & 1.704 & 1.068-2.719 & 0.025\end{array}$

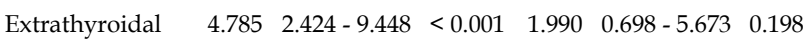

extension

$\begin{array}{lllllll}\text { Multifocality } \quad 2.124 & 1.096-4.115 & 0.026 & 0.558 & 0.171-1.825 & 0.335\end{array}$

$\begin{array}{lllllll}\text { Lymphovascular } & 4.048 & 2.092-7.835<0.001 & 1.856 & 0.589-5.848 & 0.291\end{array}$

invasion

Lymph node $\quad 4.800 \quad 2.383-9.671<0.001 \quad 4.678 \quad 1.238-\quad 0.023$

metastasis

BRAF mutation $\quad 0.686 \quad 0.277-1.696 \quad 0.414$

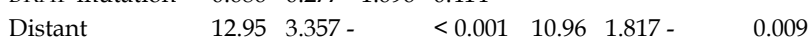

$\begin{array}{lllll}\text { metastasis } & 1 & 49.962 & 5 & 66.161\end{array}$

Preoperative $\quad 1.293 \quad 0.868-1.926 \quad 0.206$

NLR $\dagger$

Increased NLR ${ }^{\ddagger} 13.68 \quad 3.454-\quad<0.001 \quad 18.66 \quad 3.260-$

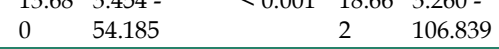

0.001

Note: Variables shown to be significant in the univariate analysis were included in the multivariate analysis. ${ }^{\dagger}$ Modeled as a continuous variable. ${ }^{\ddagger}$ As a binary variable (increased NLR versus stable/decreased NLR). Abbreviation: NLR, neutrophil-to-lymphocyte ratio

Depending on the response to therapy, the initial staging evaluation may not be as accurate as dynamic risk stratification in predicting patient outcome.
Similarly, the dynamics of inflammatory biomarkers may be of more prognostic value than the pretreatment level. The importance of the changes in NLR after treatment, as seen in this study, is not unique to thyroid cancer. Peng et al. demonstrated that an increased NLR, but not preoperative or postoperative NLR, was an independent predictor of overall and recurrence-free survival in patients with small hepatocellular carcinoma [23]. The association of the changes in NLR with survival has also been reported in other types of malignancy, such as glioblastoma, lung, and gastric cancers [24-26].

Interestingly, the dynamics of inflammatory biomarkers can also be exploited as useful surrogates for response to various therapies. An early decline of NLR was associated with favorable outcomes during targeted therapy or immune checkpoint blockade for metastatic renal cell carcinoma [27, 28]. By contrast, Saied and colleagues demonstrated that increased NLR levels were associated with poor responses following hepatic artery infusions of chimeric antigen receptor-modified $\mathrm{T}$ cells for unresectable liver metastases [29]. In borderline resectable pancreatic cancer, an increased NLR after neoadjuvant therapy was associated with worse survival [10]. The increase in NLR following treatment may reflect the disease progression or indicate an inadequate modulation of the host immune response [30]. Taken together, the change in NLR before and after treatment is not only a prognostic but a predictive indicator which may tailor follow-up and future therapeutic approaches.

We observed a small but significant reduction in NLR among patients with an excellent response. Strikingly, the post-treatment NLR levels in these patients were even lower than the postoperative NLR levels in patients who underwent thyroidectomy for benign thyroid lesions. A reasonable interpretation of these results is that the decline in NLR was likely caused by the effects of RAI therapy. Asymptomatic drops in white blood cell count are frequently seen with usual doses of RAI and may persist for one year $[31,32]$. Nonetheless, serious bone marrow suppression is rare and occurs exceptionally in patients who had both RAI therapy and external radiation therapy to bone metastases. Lymphocytes appear to be more susceptible to RAI than do neutrophils [33]. As such, one would expect a rise in NLR following RAI therapy. In this respect, Demir and collaborators demonstrated that the NLR level significantly increased two months after RAI and returned nearly to baseline by six months after RAI [34]. The timing of obtaining the follow-up NLR data in this study was 6 to 18 months post treatment and prior to TSH stimulation to verify the absence of disease. Based on our results, we speculate that for 
patients on thyroid hormone therapy who have an undetectable $\mathrm{Tg}$ level with the use of a sensitive $\mathrm{Tg}$ assay and a stable or decreased NLR at follow-up, TSH stimulation testing may be no longer necessary.

Limitations of this study include its retrospective design. About one-third of our patients were excluded from the analysis because they did not have available follow-up NLR data during the desired time frame. The number of patients with an incomplete response to therapy is relatively small $(n=34)$. The rule of thumb for logistic and Cox models recommends a minimum of 10 outcome events per predictor variable (EPV) to avoid the risk of overfitting. However, the validity of this thumb rule has been questioned, and the rule of $10 \mathrm{EPV}$ could be relaxed, depending on the research question [35]. The application of bootstrap sampling procedure in our regression analysis gave similar results (data not shown).

Furthermore, our study was limited by its relatively short follow-up period, which restricts the ability to make meaningful conclusions with respect to recurrence or mortality. A power calculation suggested that a sample size of 360 to 800 patients with a follow-up of 6 to 10 years is required for the recurrence analysis in papillary thyroid cancer [36]. Nonetheless, this study supports the hypothesis that the magnitude of systemic inflammation reflects the disease burden and the status of the response to therapy in differentiated thyroid cancer. The findings reported herein offer a number of potentially fruitful avenues for future research.

In conclusion, our present study found that an increase in systemic inflammation as measured by NLR is independently associated with an incomplete response to therapy in differentiated thyroid cancer. NLR dynamics may provide a readily available biomarker to guide individualized patient care.

\section{Acknowledgements}

This work was supported in part by research grants from the Ministry of Science and Technology of Taiwan (MOST-106-2314-B-715-008-MY3) and Mackay Medical College (RD1070106).

\section{Ethics approval}

The study (17MMHIS035) has been reviewed and approved by the Institutional Review Board of MacKay Memorial Hospital.

\section{Competing Interests}

The authors have declared that no competing interest exists.

\section{References}

1. Hanahan D, Weinberg RA. Hallmarks of cancer: the next generation. Cell. 2011; 144: 646-674.
2. Diakos CI, Charles KA, McMillan DC, Clarke SJ. Cancer-related inflammation and treatment effectiveness. Lancet Oncol. 2014; 15: e493-e503.

3. Li X, Dai D, Chen B, Tang H, Xie X, Wei W. The value of neutrophil-to-lymphocyte ratio for response and prognostic effect of neoadjuvant chemotherapy in solid tumors: A systematic review and meta-analysis. J Cancer. 2018; 9: 861-871.

4. Templeton AJ, McNamara MG, Seruga B, Vera-Badillo FE, Aneja P, Ocana A, Leibowitz-Amit R, Sonpavde G, Knox JJ, Tran B, Tannock IF, Amir E. Prognostic role of neutrophil-to-lymphocyte ratio in solid tumors: a systematic review and meta-analysis. J Natl Cancer Inst. 2014; 106: dju124.

5. Liu CL, Lee JJ, Liu TP, Chang YC, Hsu YC, Cheng SP. Blood neutrophil-to-lymphocyte ratio correlates with tumor size in patients with differentiated thyroid cancer. J Surg Oncol. 2013; 107: 493-497.

6. Lang BH, Ng CP, Au KB, Wong KP, Wong KK, Wan KY. Does preoperative neutrophil lymphocyte ratio predict risk of recurrence and occult central nodal metastasis in papillary thyroid carcinoma? World J Surg. 2014; 38: 2605-2612.

7. Kim JY, Park T, Jeong SH, Jeong CY, Ju YT, Lee YJ, Hong SC, Ha WS, Choi SK, Jung EJ. Prognostic importance of baseline neutrophil to lymphocyte ratio in patients with advanced papillary thyroid carcinomas. Endocrine. 2014; 46: 526-531.

8. Krajewska J, Chmielik E, Jarzab B. Dynamic risk stratification in the follow-up of thyroid cancer: what is still to be discovered in 2017? Endocr Relat Cancer. 2017; 24: R387-R402.

9. Yang PS, Liu CL, Liu TP, Chen HH, Wu CJ, Cheng SP. Parathyroidectomy decreases neutrophil-to-lymphocyte and platelet-to-lymphocyte ratios. J Surg Res. 2018; 224: 169-175.

10. Glazer ES, Rashid OM, Pimiento JM, Hodul PJ, Malafa MP. Increased neutrophil-to-lymphocyte ratio after neoadjuvant therapy is associated with worse survival after resection of borderline resectable pancreatic ductal adenocarcinoma. Surgery. 2016; 160: 1288-1293.

11. Cheng SP, Chien MN, Wang TY, Lee JJ, Lee CC, Liu CL. Reconsideration of tumor size threshold for total thyroidectomy in differentiated thyroid cancer. Surgery 2018; 164: 504-510.

12. Lee JJ, Wang TY, Liu CL, Chien MN, Chen MJ, Hsu YC, Leung CH, Cheng SP. Dipeptidyl peptidase IV as a prognostic marker and therapeutic target in papillary thyroid carcinoma. J Clin Endocrinol Metab. 2017; 102: 2930-2940.

13. Cheng SP, Hsu YC, Liu CL, Liu TP, Chien MN, Wang TY, Lee JJ. Significance of allelic percentage of BRAF c.1799T > A (V600E) mutation in papillary thyroid carcinoma. Ann Surg Oncol. 2014; 21 Suppl 4: S619-S626.

14. Haugen BR, Alexander EK, Bible KC, Doherty GM, Mandel SJ, Nikiforov YE, Pacini F, Randolph GW, Sawka AM, Schlumberger M, Schuff KG, Sherman SI, Sosa JA, Steward DL, Tuttle RM, Wartofsky L. 2015 American Thyroid Association Management Guidelines for Adult Patients with Thyroid Nodules and Differentiated Thyroid Cancer: The American Thyroid Association Guidelines Task Force on Thyroid Nodules and Differentiated Thyroid Cancer. Thyroid. 2016; 26: 1-133.

15. Momesso DP, Vaisman F, Yang SP, Bulzico DA, Corbo R, Vaisman M, Tuttle RM. Dynamic risk stratification in patients with differentiated thyroid cancer treated without radioactive iodine. J Clin Endocrinol Metab. 2016; 101: 2692-2700.

16. Hsu CC, Wang YM, Huang CR, Sun FJ, Lin JP, Yip PK, Liu SI. Sustained benefit of a psycho-educational training program for dementia caregivers in Taiwan. Int J Gerontol. 2017; 11: 31-35.

17. Pusztaszeri MP, Faquin WC, Sadow PM. Tumor-associated inflammatory cells in thyroid carcinomas. Surg Pathol Clin. 2014; 7: 501-514.

18. Gentles AJ, Newman AM, Liu CL, Bratman SV, Feng W, Kim D, Nair VS, Xu Y, Khuong A, Hoang CD, Diehn M, West RB, Plevritis SK, Alizadeh AA. The prognostic landscape of genes and infiltrating immune cells across human cancers. Nat Med. 2015; 21: 938-945.

19. Hsu YC, Liu CL, Yang PS, Tsai CH, Lee JJ, Cheng SP. Interaction of age at diagnosis with transcriptional profiling in papillary thyroid cancer. World J Surg. 2016; 40: 2922-2929.

20. Cheng SP, Chen MJ, Chien MN, Lin CH, Lee JJ, Liu CL. Overexpression of teneurin transmembrane protein 1 is a potential marker of disease progression in papillary thyroid carcinoma. Clin Exp Med. 2017; 17: 555-564.

21. Peters SB, Abdelatif O, LiVolsi VA. Intraepithelial neutrophils in thyroid fine-needle aspiration: a portent of aggressive thyroid cancer? Endocr Pathol. 1996; 7: 47-54.

22. Liu JF, Ba L, Lv H, Lv D, Du JT, Jing XM, Yang NJ, Wang SX, Li C, Li XX. Association between neutrophil-to-lymphocyte ratio and differentiated thyroid cancer: a meta-analysis. Sci Rep. 2016; 6: 38551.

23. Peng W, Li C, Wen TF, Yan LN, Li B, Wang WT, Yang JY, Xu MQ. Neutrophil to lymphocyte ratio changes predict small hepatocellular carcinoma survival. J Surg Res. 2014; 192: 402-408.

24. Mason M, Maurice C, McNamara MG, Tieu MT, Lwin Z, Millar BA, Menard C, Laperriere N, Milosevic M, Atenafu EG, Mason W, Chung C. Neutrophil-lymphocyte ratio dynamics during concurrent chemo-radiotherapy for glioblastoma is an independent predictor for overall survival. J Neurooncol. 2017; 132: 463-471.

25. Derman BA, Macklis JN, Azeem MS, Sayidine S, Basu S, Batus M, Esmail F, Borgia JA, Bonomi P, Fidler MJ. Relationships between longitudinal neutrophil to lymphocyte ratios, body weight changes, and overall survival in patients with non-small cell lung cancer. BMC Cancer. 2017; 17: 141. 
26. Min KW, Kwon MJ, Kim DH, Son BK, Kim EK, Oh YH, Wi YC. Persistent elevation of postoperative neutrophil-to-lymphocyte ratio: A better predictor of survival in gastric cancer than elevated preoperative neutrophil-to-lymphocyte ratio. Sci Rep. 2017; 7: 13967.

27. Templeton AJ, Knox JJ, Lin X, Simantov R, Xie W, Lawrence N, Broom R, Fay AP, Rini B, Donskov F, Bjarnason GA, Smoragiewicz M, Kollmannsberger C, Kanesvaran R, Alimohamed N, Hermanns T, Wells JC, Amir E, Choueiri TK, Heng DY. Change in neutrophil-to-lymphocyte ratio in response to targeted therapy for metastatic renal cell carcinoma as a prognosticator and biomarker of efficacy. Eur Urol. 2016; 70: 358-364.

28. Lalani AA, Xie W, Martini DJ, Steinharter JA, Norton CK, Krajewski KM, Duquette A, Bosse D, Bellmunt J, Van Allen EM, McGregor BA, Creighton CJ, Harshman LC, Choueiri TK. Change in neutrophil-to-lymphocyte ratio (NLR) in response to immune checkpoint blockade for metastatic renal cell carcinoma. J Immunother Cancer. 2018; 6: 5 .

29. Saied A, Licata L, Burga RA, Thorn M, McCormack E, Stainken BF, Assanah EO, Khare PD, Davies R, Espat NJ, Junghans RP, Katz SC Neutrophil:lymphocyte ratios and serum cytokine changes after hepatic artery chimeric antigen receptor-modified T-cell infusions for liver metastases. Cancer Gene Ther. 2014; 21: 457-462.

30. Cheng SP. Inflammatory and immune responses to surgery in the elderly: smoke or fire? Int J Gerontol. 2017; 11: 55.

31. Molinaro E, Leboeuf R, Shue B, Martorella AJ, Fleisher M, Larson S, Tuttle RM. Mild decreases in white blood cell and platelet counts are present one year after radioactive iodine remnant ablation. Thyroid. 2009; 19: 1035-1041.

32. Prinsen HT, Klein Hesselink EN, Brouwers AH, Plukker JT, Sluiter WJ, van der Horst-Schrivers AN, van Imhoff GW, Links TP. Bone marrow function after (131)I therapy in patients with differentiated thyroid carcinoma. J Clin Endocrinol Metab. 2015; 100: 3911-3917.

33. Bikas A, Schneider M, Desale S, Atkins F, Mete M, Burman KD, Wartofsky L, Van Nostrand D. Effects of dosimetrically guided I-131 therapy on hematopoiesis in patients with differentiated thyroid cancer. J Clin Endocrinol Metab. 2016; 101: 1762-1769.

34. Demir Y, Ucler R, Surucu E, Turan M, Balli Z, Sengoz T. Temporary changes in neutrophil-to-lymphocyte, platelet-to-lymphocyte ratios, and mean platelet volume reflecting the inflammatory process after radioiodine therapy. Nucl Med Commun. 2016; 37: 393-398.

35. Vittinghoff $\mathrm{E}, \mathrm{McC}$ culloch $\mathrm{CE}$. Relaxing the rule of ten events per variable in logistic and Cox regression. Am J Epidemiol. 2007; 165: 710-718.

36. Udelsman R, Lakatos E, Ladenson P. Optimal surgery for papillary thyroid carcinoma. World J Surg. 1996; 20: 88-93. 